\title{
SU ALCUNE ANOMALIE DELLA FREQUENZA CRITICA IONOSFERICA DURANTE UN'ECLISSE DI SOLE
}

\author{
Guglelmo Zanotelli
}

Le eclissi di sole offrono alcune possibilità di studiare l'andamento della concentrazione degli elettroni liberi nella ionosfera in corrispondenza alle variazioni dell'agente ionizzante solare. Queste possibilità furono già nel passato messe a profitto da diversi autori, specie per lo studio dello strato $F 2$ che è quello per cui permangono ancor oggi le maggiori incertezze circa la correlazione degli elementi interessati. $\mathrm{Ci}$ sembra perciò non privo di interesse di apportare con il presente lavoro un contriluto alla questione, esponendo $\mathrm{i}$ risultati dei sondagrgi ionosferici eseguiti presso la sede dell'Istituto Nazionale di Geofisica in Roma nel corso dell'eclisse di sole del 25 febbraio 1952.

In Roma l'eclisse si presentava come molto parziale, di grandezza 0.27 in diametri al suolo, e 0,21 circa alla quota di $250 \mathrm{~km}$, corrispondente ad una occultazione massima di poco più di un decimo della superficie solare. Purtuttavia il suo influsso si è rivelato non trascurabile nelle registrazioni ionosferiche, sì da permetterci di dedurre al. cune considerazioni sull'origine del ritardo con cui la diminuzione di concentrazione elettronica si presenta rispetto alla fase massima dell'eclisse, e iell'apparente variazione del coefficiente di ricombinazione nel corso della stessa.

L'apparecchiatura radioelettrica usata esplorava automaticamente ncl giro di $20^{\prime \prime}$ la gamma di frequenze comprese fra 1,7 e $18 \mathrm{Mc}$, e registrava fotograficamente gli echi ionosferici. I valori delle frequenze critiche $f$ dedotte dalle registrazioni del giorno dell'eclisse sono riportate in funzione del tempo $t$ nella fig. 1 per lo strato $F 2$ che $\grave{\mathrm{e}}$ quello che a noi particolarmente interessa, mentre con la linea trattegviata si è indicato l'andamento medio della frequenza critica $f_{\mathrm{o}}$ ricavata dalle registrazioni dei giorni immediatamente precedenti e se«nenti quello dell'eclisse.

Siccome ai fini dell'interpretazione interessa osservare non tanto l'andamento della frequenza critica per se stessa nel corso dell'eclisse, quanto in relazione ai valori medi della stessa nei giorni precedenti 
e seguenti, si è costruito il diagramma (fig. 2) dell'andamento della funzione $\left(f / f_{0}\right)^{2}$; stante la proporzionalità della concentrazione elettronica ionosferica al quadrato della frequenza critica, tale funzione equivale al rapporto $n / n_{0}$ fra le rispettive concentrazioni elettroniche $n$ al momento dell'eclisse, ed $n_{0}$ media nei gioni precedenti e seguenti. Con questo accorgimento si sono praticamente depurati i risultati dall'influenza della variabile altezza del sole per le varie ore della giornata. La curva tratteggiata nella stessa fig. 2 rappresenta l'area solare scoperta nel corso dell'eclisse in percento dell'area totale del disco solare, per la quota di $250 \mathrm{~km}$.

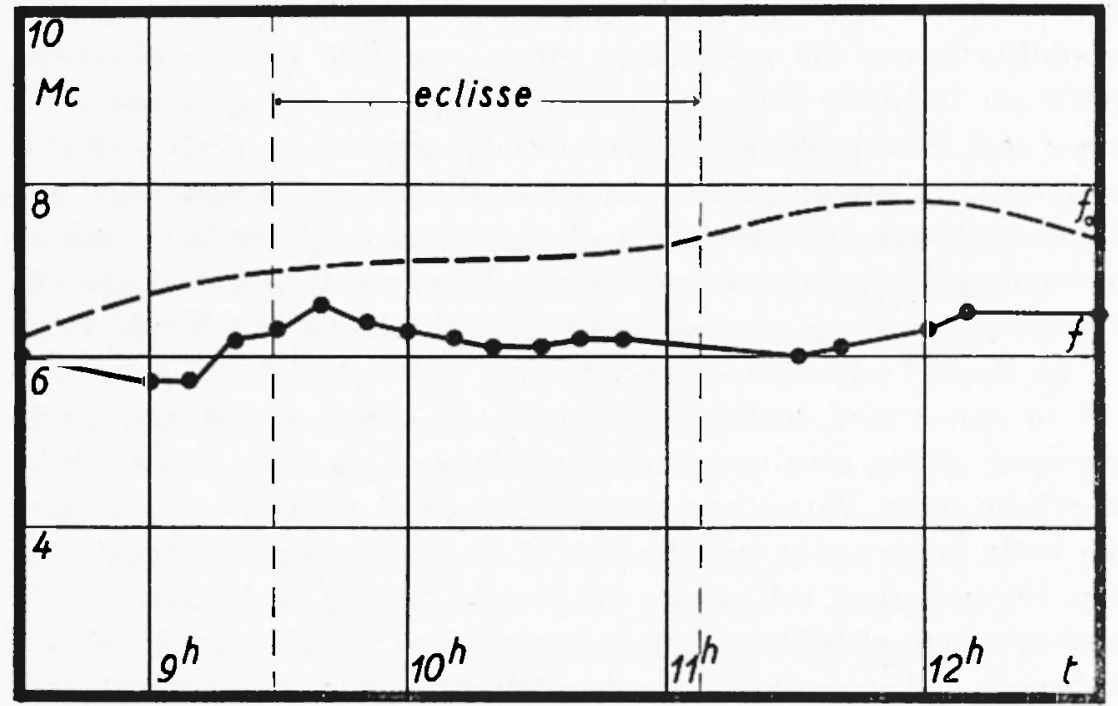

Fig. 1 - La frequenza critica $f$ durante l'eclisse di sole in confronto con quella $f_{n}$ media nei giorni precedenti e seguenti reclisse.

Dall'esame della fig. 2 risulta che la concentrazione elettronica scende al disotto del valore medio già un paio d'ore prima dell'inizio dell'eclisse, e segna un minimo netto circa $20^{\prime}$ avanti il primo contatto, con una altezza virtuale di $230 \mathrm{~km}$. Un nuovo minimo si riscontra in corrispondenza alla fase massima dell'eclisse, ed un altro infine sul finire di questa in una posizione che però non può essere determinata con esattezza per la mancanza di alcuni punti della registrazione, ma localizzabile con certezza poco oltre l'ultimo contatto. L'altezza virtuale in corrispondenza a questi altri minimi di frequenza critica fu di $250 \mathrm{~km}$. 
Se ci riferiamo alla equazione che lega la concentrazione elettronica $n$ con il numero $q$ di elettroni prodotti per unità di volume e di tempo, e con quello $r$ rispettivamente di elettroni eliminati,

$$
\frac{d n}{d t}=q-r,
$$

essendo in corrispondenza ad un minimo $d n / d t=0$ deve aversi per l'equilibrio

$$
q=r
$$

Esaminando in fig. 1 la curva dell'andamento medio della frequenza critica nei giorni non di eclisse, si riscontra che in corrispondenza all'ora della fase massima tale curva decorre quasi orizzontale, ed ivi potrà supporsi che per la corrispondente concentrazione elettronica sia $d n_{0} / d t=0 ;$ quindi

$$
\boldsymbol{q}_{o}=\boldsymbol{r}_{o}
$$

essendosi contrassegnate con l'indice zero le grandezze in assenza di eclisse.

Viene generalmente ammesso che il numero $q$ di elettroni prodotti sia proporzionale all'area scoperta $S$ del disco solare, e che gli elettroni siano soltratti alla ionosfera in virtù del processo di ricombinazione, per cui $r=\alpha n^{2}$, ove $\alpha$ è il coefficiente di ricombinazione. Dividendo membro a membro le [2] e [3] si ottiene allora

$$
\frac{q}{q_{0}}=\frac{r}{r_{\mathbf{0}}}
$$

cioè

$$
\frac{S}{S_{0}}=\frac{\alpha n^{2}}{\alpha_{0} n_{0}^{2}}=\frac{\alpha f^{4}}{\alpha_{0} \gamma_{0}^{4}}
$$

Per la fase massima della nostra eclisse è circa $S / S_{o}=0,89$ e corrispondentemente $n / n_{0}=0,73$ : per la precedente dovrebbe ammettersi che forse $\alpha / \alpha_{0}=1,7$, cioè che il coefficiente di ricombinazione fosse aumentato rispetto al normale di circa il $70 \%$. È bensi vero che si potrebbe introdurre come causa di diminuzione della concentrazione elettronica oltre il processo di ricombinazione anche la cattura 
degli elettroni da parte di molecole o di atomi (1); ciò equivarrebbe a porre $r=u n^{2}+b n$. Tuttavia anche in questo caso si dovrebbero supporre diversi i valori dei coefficienti $\alpha, b$, in presenza od in assenza di eclisse, per giustificare il valore trovato 0,73 .

Non mancano certo le ragioni per spiegare la variazione di un coefficiente che in realtà si dovrebbe supporre costante, quale quello di ricombinazione. Tuttavia le considerazioni che ora svolgeremo circa

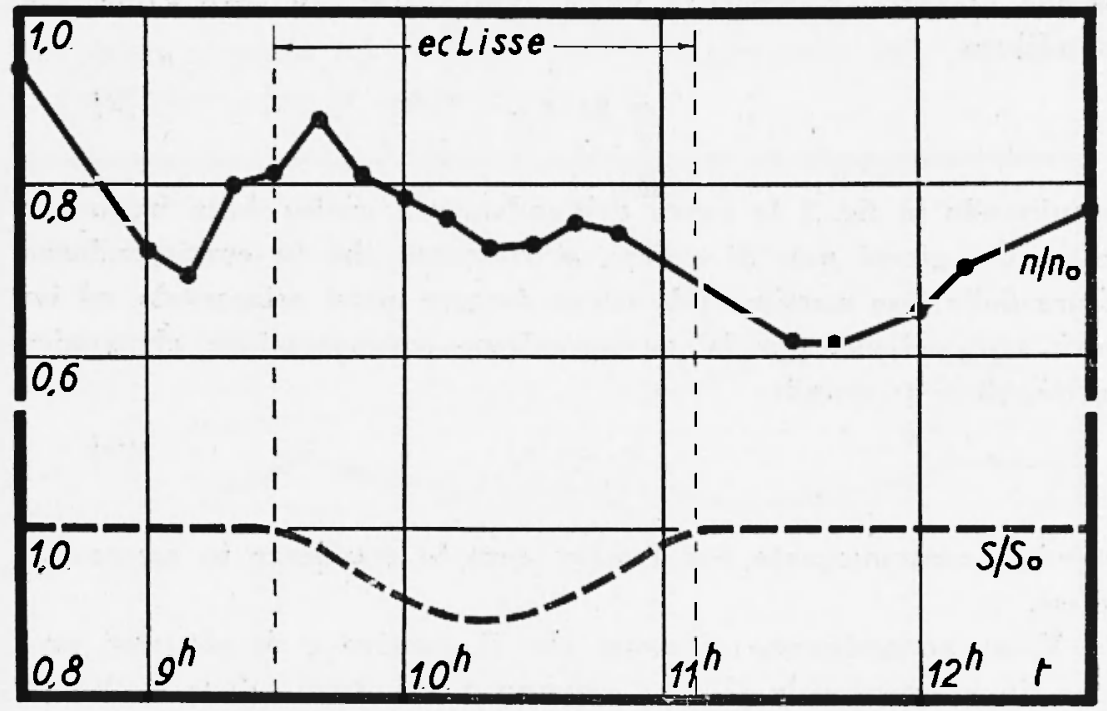

Fig. 2 - Il rapporto fra la concentrazione elettronica $n$ durante l'eclisse ed $n_{\text {s }}$ media nei giorni precedenti e seguenti in confronto alla percentuale di superficio soline scoperta.

il minimo che presenta la funzione $n / n_{0}$ in corrispondenza al finire dell'eclisse sembrano indurci a rivolgere la nostra attenzione piuttosto alla funzione $q$ che non alla $r$. Infatti si tratta in quest'ultimo caso di interpretare non solo il significato della diminuzione della concentrazione elettronica che si presenta al termine dell'eclisse, ma anche di spiegare l'origine di un ritardo così notevole rispetto al massimo occultamento della superficie solare. Si noti che tale ritardo si riscontra oltre che nelle nostre registrazioni, anche nei rilevamenti ionosferici di altri autori $\left({ }^{2}\right)\left({ }^{3}\right)$ in corrispondenza di eclissi solari.

Pure nel punto di minimo che ora consideriamo deve verificarsi per l'equilibrio, almeno approssimativamente la [4]; se si volesse però assumere ancora il numero degli elettroni prodotti come proporzionale alla superficie solare scoperta, si dovrebbe prendere ormai uguale ad 
uno il primo membro di tale relazione, e quindi, ammettendo agente la sola ricombinazione, essendo ora $n / n_{0}$ uguale a circa 0,6 , si perverrebbe ad un rapporto $\alpha / \alpha_{0}=2,7$ circa. Poiché l'altezza virtuale dello strato $F 2$ desunta dalle registrazioni in corrispondenza a questo minimo coincide con quella per il minimo centrale, a questo elemento non può pertanto attribuirsi la forte variazione del coefficiente di ricombinazione.

Per renderci ragione di come possano svolgersi i fatti, riflettiamo che le cause di eliminazione degli elettroni dalla ionosfera sono in generale interne a questa, anzi locali e ristrette attorno al punto considerato, come appunto la ricombinazione degli elettroni, la cattura di questi da parte di molecole o da atomi, e simili. Quale influsso potrebbe dunque avere su queste cause locali l'eclisse dopo un'ora circa dalla sua fase massima, e quando la superficie solare è di nuovo completamente scoperta? In qual modo potrebbe giustificarsi la forte variazione del coefficiente di ricombinazione per $i$ due minimi ora esaminati con un ipotetico effetto dell'eclisse?

Sembra senz'altro più logico ammettere che il ritardo con cui si presenta la variazione della concentrazione elettronica sia da imputare alla stessa causa da cui la $q$ deriva, causa esterna alla ionosfera ed estranea perciò ad ogni circostanza locale di questa. Ciò equivale a supporre la $q / q_{0}$ non coincidente con la $S / S_{0} ;$ o quanto meno ad identificarla con una $S / S_{\text {o }}$ ritardata di un conveniente intervallo di tempo: tuttavia se quest'ultima ammissione può valere a spiegare il minimo che si riscontra sul finire dell'eclisse, di alcun ausilio ci sarebbe per l'interpretazione di quel minimo che nelle nostre registrazioni appare ancor prima dell'inizio di quella.

Siamo perciò naturalmente orientati a mettere in relazione la $q$ con qualche causa, capace di indurre la produzione di elettroni ionosferici, localizzata in zone esterne alla parte visibile del disco solare: l'occultamento di tali zone da parte della luna potrebbe essere la causa dei due minimi estremi; potrebbe altresì contribuire, insieme con l'occultamento di una porzione della superficie solare visibile, al minimo centrale, rendendo ragione del valore sperimentalmente riscontrato nella diminuzione di concentrazione elettronica, senza ricorrere ad un fittizio aumento del coefficiente di ricombinazione.

Questa conclusione cui siamo pervenuti ragionando sugli elementi tratti dalle nostre registrazioni viene corroborata dalle circostanze che si presentarono per le eclissi solari del 4 e 5 febbraio 1943 , $1^{\circ}$ agosto 1943 e 25 gennaio 1944; tali eclissi furono studiate da Wells e 
Shapley $\left(^{2}\right)$ e presentarono minimi della frcquenza critica all'inizio e sul finire dell'eclisse, che gli stessi autori ritennero poter essere messi in relazione ad una concorrente attività solare, e specialmente a zone luminose della corona.

Roma - Istituto Nazionale di Geofisica - Giugno 1953.

\section{RIASSUNTO}

Si espongono $i$ risultati dei sondaggi ionosferici eseguiti in Romn durante l'eclisse parziale di sole del 25 febbraio 1952, dai quali risul. tarono tre minimi della concentrazione elettronica, uno prima dell'inizio dell'eclisse, uno in corrispondenza alla fase massima ed uno sul finire dell'eclisse stessa. Si mostra che ammettendo la causa dei due minimi estremi come localizzata in zone esterne alla parte visibile del disco solare, si può dar ragione dell'altrimenti inesplicabile ritardo dell'ultimo minimo rispetto all'eclisse visibile, e si può interpretare nel contempo la forte variazione apparente del coefficiente di ricombinazione per gli elettroni ionosferici.

\section{SUMMARY}

The results of the ionospheric soundings carried out in Rome during the partial eclipse of the sun of February 25, 1952 are presented here. Three minima vere found in the electronic concentration; one before the biginning of the eclipse, one for the maximum phase, and one at the end of the eclipse. It is shou'n, admitting that the cause of the two extreme minima is to be found in the external zone of the visible part of the solar dish, that one can explain the otherwise inexplicable retard of the last minimum with respect to the visible eclipse, and one can interpret, at the same time, strong apparent variation in the coefficient of recombination of the ionospheric electrons.

\section{BIBLIOGRAFIA}

(1) Cfr. S. N. Gноsн, Proc. Nat. Inst. Sci. of India, 10, 333, 1944.

(2) H. W. Wells e A. H. Shapley, Terr, Magn. 51, 401, 1946.

(3) H. W. Wells, Journ. Geoph. Res. 57, 291, 1952. 